Archives de sciences sociales des religions

133 | janvier - mars 2006

Catholicismes

\title{
Médiation et institution catholique
}

Jacques Palard

\section{OpenEdition}

Journals

Édition électronique

URL : http://journals.openedition.org/assr/3358

DOI : $10.4000 /$ assr.3358

ISSN : 1777-5825

Éditeur

Éditions de l'EHESS

Édition imprimée

Date de publication : 1 mars 2006

ISBN : 2-7132-2046-7

ISSN : 0335-5985

Référence électronique

Jacques Palard, «Médiation et institution catholique », Archives de sciences sociales des religions [En ligne], 133 | janvier - mars 2006, mis en ligne le 04 décembre 2013, consulté le 19 avril 2019. URL http://journals.openedition.org/assr/3358 ; DOI : 10.4000/assr.3358 


\section{Jacques Palard}

\section{Médiation et institution catholique}

Les pratiques de médiation connaissent un fort développement dans l'ensemble des secteurs de la vie sociale et politique des sociétés occidentales contemporaines ${ }^{1}$ : la famille, l'institution judiciaire, la gouvernance urbaine, l'entreprise, l'école, les rapports entre l'État et les citoyens, les relations internationales... La France n'a pas échappé à ce processus : la création du Médiateur de la République en 1973, des conciliateurs en 1978 ou des médiations pénales ou sociales en 1985 illustre et légitime un phénomène qui traduit une transformation en profondeur des modalités de gestion des conflits. Ce développement de nouvelles procédures, qui sont autant d'outils de pacification sociale selon un mode négocié, n'est évidemment pas sans lien avec la crise des mécanismes traditionnels de gestion et de régulation des rapports sociaux. J.-P. Bonafé-Schmitt souligne ainsi à propos de la création des « boutiques de droit » que ces nouvelles instances, qui remplissent une fonction d'interface, offrent un modèle de règlement des litiges fondé à la fois sur la décentralisation, la déprofessionnalisation et la délégalisation et autorisent par là une réappropriation par les citoyens des modes de gestion des conflits ${ }^{2}$.

L'un des observateurs les plus avertis de ce type d'innovations sociales, luimême anthropologue du droit, note que " la médiation, comme cadre spécifique, repose sur des manières de faire, des procédés et des procédures qui sont encore mal élucidés tant du point de vue théorique que pratique ». Par voie de conséquence, il estime qu' "approfondir de telles connaissances en partant d'observations de terrains, construire de nouveaux modèles (comme représentations à la fois simplifiées et globales) des phénomènes considérés et comparer ces différentes interprétations sont les tâches que nous devons assigner à la recherche

1. Cf. Peter L. Berger, dir., The Limits of Social Conflict. Conflict and Mediation in Pluralist Societies (A Report of the Bertelsmann Foundation to the Club of Rome), Boulder, Westview Press, 1998 ; Edward W. SCHWERIN, Mediation, Citizen Empowerment and Transformational Politics, Westport, Praeger, 1995 ; W. Robert Lovan, Michael MurRay, Ron SHAFFer, eds, Participatory Governance: Planning, Conflict Mediation and Public Decision-making in Civil Society, Aldershot, Ashgate, 2004 ; CONSEIL DE L'EUROPE, Les solutions alternatives aux litiges entre les autorités administratives et les personnes privées : conciliation, médiation et arbitrage, Strasbourg, Éditions du Conseil de l'Europe, 2000.

2. Jean-Pierre BonAfé-SchmitT, "La médiation, un nouveau mode de régulation sociale ", Histoires de développement, $\mathrm{n}^{\circ}$ 20, décembre 1992, p. 35. 
interdisciplinaire en sciences sociales ${ }^{3}$. Sans évidemment faire sien l'ensemble d'une telle entreprise analytique, cette étude cherchera à éclairer l'une des dimensions possibles de ce projet de recherche interdisciplinaire en poussant l'investigation du côté des modèles sociaux inspirés du catholicisme. Jacques Faget parle de la culture de la médiation, notamment dans ses rapports avec l'institution judiciaire, comme d'une « culture sans origine qui se construit lentement, imperceptiblement au fil des impensés et des impensables de l'existence ${ }^{4}$. Le propos est au contraire ici de rechercher - du côté des pratiques et des discours de l'institution catholique - quelques-uns des substrats de cette culture ${ }^{5}$.

Un tel rapprochement entre médiation et catholicisme ne va pas sans un apparent paradoxe. En effet, dans la sphère religieuse, c'est le régime de l'hétéronomie qui prévaut; dans celle de la médiation, prime au contraire, par principe même, la négociation entre acteurs qui se pensent libres et égaux. Là, les croyants se situent dans l'ordre d'une vérité révélée à caractère absolu et, par définition, intangible ; ici, les acteurs œuvrent à faire advenir une vérité négociée et contingente par voie d'échange, d'arbitrage et de co-production. Là, c'est le rapport à la transcendance qui, à coup sûr, domine ; ici, se développe le libre jeu des rapports sociaux immanents.

Pourtant, à observer les conditions d'émergence de l'exercice de la médiation, les orientations philosophiques et religieuses de ses initiateurs et de ses promoteurs, le système de valeurs qui en légitime la mise en œuvre, on se prend à penser que des liens sinon de filiation du moins d'affinité ou de connivence se nouent entre cette pratique sociale protéiforme et des orientations socioreligieuses prônées par le christianisme : la notion de réconciliation, la restauration du lien, l'utopie de la communion... L'analyse des notions de subsidiarité et de corps intermédiaires dans la doctrine sociale de l'Église catholique (1) et celle des nouvelles formes d'investissement de l'espace public par des acteurs religieux (2) permettent d'avancer sur cette voie. On est en effet fondé à estimer que ces orientations à la fois discursives et pratiques prennent en compte les trois composantes que la plupart des observateurs s'accordent à reconnaître comme fondamentales dans toute définition de la médiation : un conflit, l'intervention d'un tiers (si possible indépendant, impartial et sans pouvoir ${ }^{6}$ ), enfin, un processus d'échanges verbalisés ordonné à la recherche d'une solution par consensus.

3. Étienne Le Roy, "La médiation mode d'emploi », Droit et société, 29, 1995, p. 54.

4. Jacques FAGET, La médiation. Essai de politique pénale, Ramonville, Érès, 1997, p. 9.

5. Dans ce domaine du droit et de la justice, Jean-Pierre BonAFÉ-SCHMITT montre en quoi des mouvements religieux, comme celui des Quakers, ont fortement marqué, sur le plan idéologique, les mouvements de médiation aux États-Unis, dont le modèle de «justice restaurative » ou "réparative " repose sur deux principes de base : l'idée de réconciliation et l'intervention de la communauté dans la résolution des conflits ("Le mouvement "Victim-Offender Mediation" : l'exemple du Minnesota Citizen Council on Crime and Justice ", Droit et Société, 29, 1995, p. 57-77.

6. «Sans pouvoir décisionnel en tant que tel, sans mandat d'une instance supérieure au nom de laquelle il parlerait, le médiateur travaille sans armes, à mains nues. Son seul outil est cette capacité d'analyser exactement des situations et de découvrir, avec les "deux", la brèche à travers 


\section{Subsidiarité et corps intermédiaires dans la doctrine sociale de l'Église catholique}

La conception catholique de l'ordre social met en avant deux principes de base qui sont commandés par le postulat d'une possible harmonie sociale. En premier lieu, il n'y a pas d'opposition sociale ou de conflit qui soit irréductible, ce qui invalide fondamentalement une lecture marxiste de l'histoire en termes de lutte de classes. Par ailleurs, l'État n'a pas à intervenir dans des domaines et à des niveaux d'action où des intervenants infra-étatiques ou sociaux paraissent constituer des opérateurs au moins aussi qualifiés et compétents; il y a dans ce principe dit de «subsidiarité » la traduction d'une philosophie politique qui n'est pas complètement dénuée d'une stratégie visant à circonscrire la sphère d'action de l'État, instance qui demeure implicitement le principal concurrent de l'Église dans la maîtrise des rapports sociaux et l'inculcation de valeurs.

L'enseignement pontifical est ici de toute première importance, à commencer par celui de Pie XI et de son encyclique Quadragesimo Anno du 15 mai 1931 (qui célèbre le "quarantième anniversaire " de l'encyclique Rerum Novarum de Léon XIII, de 1891, et qui comporte des éléments de doctrine corporative, conséquence du rôle accordé à l'organisation professionnelle) :

Il est vrai sans doute, écrit le Pape, (...) que bien des choses que l'on demandait jadis à des associations de moindre envergure ne peuvent plus désormais être accomplies que par de grandes collectivités. Il n'en reste pas moins indiscutable qu'on ne saurait ni changer, ni ébranler ce principe si grave de philosophie sociale : de même qu'on ne peut enlever aux particuliers, pour les transférer à la communauté, les attributions dont ils sont capables de s'acquitter de leur seule initiative et par leurs propres moyens, ainsi ce serait commettre une injustice en même temps que troubler d'une manière dommageable l'ordre social que de retirer aux groupements d'ordre inférieur, pour les confier à une collectivité plus vaste et d'un rang plus élevé, les fonctions qu'ils sont en mesure de remplir eux-mêmes. L'objet naturel de toute intervention en matière sociale est d'aider les membres du corps social et non pas de les détruire, ni de les absorber. (...) Que les gouvernements en soient donc persuadés : plus parfaitement sera réalisé l'ordre hiérarchique des divers groupements, selon ce principe de la fonction supplétive de toute collectivité, plus grandes seront l'autorité et la puissance sociale, plus heureux et plus prospère l'état des affaires publiques.

Dans son encyclique Mater et Magistra (1961), Jean XXIII reprend in extenso le propos de Pie XI mais, tirant la leçon des corporatismes autoritaires naguère triomphants, il s'attache à promouvoir le rôle des "corps intermédiaires ${ }^{7}$ :

laquelle une certaine solution est possible " (Jean-François SIX, " Médiation et médiateurs ", Études, juillet-août 1993, p. 50).

7. Michel CREUZET en donne la définition suivante : «Les corps intermédiaires sont des groupes sociaux, des groupements humains situés entre l'individu isolé (ou la famille, cellule de base) et l'État. Ils sont constitués soit naturellement [par exemple, les corps professionnels (entreprise, métier, profession) ou locaux (commune, province)], soit par accord délibéré [lorsqu'ils résultent d'une entente entre les membres : société de pêche, compagnonnage, académie provinciale...] en vue d'atteindre une fin commune aux personnes qui les composent " (Les corps intermédiaires, Martigny, Édition des Cercles Saint-Joseph, 1964, p. 11). 
Nous estimons (...) nécessaire que les corps intermédiaires et les initiatives sociales diverses, par lesquelles surtout s'exprime et s'organise la "socialisation ", jouissent d'une autonomie efficace devant les pouvoirs publics, qu'ils poursuivent leurs intérêts spécifiques en rapport de collaboration loyale entre eux et de subordination aux exigences du bien commun ${ }^{8}$.

On rejoint par là la position d'un René Capitant, pour qui la démocratie est essentiellement la doctrine qui tend à ne considérer comme légitimes que "les obligations juridiques autonomes $"{ }^{9}$.

En ce qui concerne le "dialogue » du privé et du public, le théologien Pierre Bigo estime que " l'élaboration d'un tissu interstitiel, d'un organisme de relations qui ne repose ni sur la seule volonté des contractants, ni sur la seule volonté de l'État, est une nécessité de la société économique et de son intégration à la société globale. L'État ne doit pas empêcher la formation et l'activité de ces groupements mineurs sous son contrôle, dans les rôles et les tâches qui leur reviennent. Il doit, au contraire, les favoriser, et son rôle propre en sera grandement facilité " ${ }^{10}$. On a de cette position une illustration récente avec la déclaration, en 1999, de la Commission sociale de l'épiscopat, rendue publique sous le titre: Réhabiliter la politique. Les auteurs s'attachent à souligner ce qui leur apparaît comme une "convergence entre les valeurs de la démocratie et les sources d'inspiration de la foi chrétienne ». Ils retiennent trois points, jugés à cet égard essentiels : l'importance, précisément, des corps intermédiaires, en ce qu'ils aident à la responsabilité de tous et sont un frein au risque d'abus de pouvoir ; le principe de subsidiarité, qui demande de "laisser à l'échelon d'organisation le plus proche ce qui peut y être traité »; enfin, la reconnaissance du pluralisme, qui témoigne de la relativité et des limites des programmes politiques, lesquels ne peuvent jamais prétendre incarner toute la vérité. Cette ligne de pensée s'accorde somme toute assez bien avec la position défendue à la fin des années 1970, singulièrement au sein du Parti socialiste et autour de la revue Faire, par les « expérimentateurs

8. La définition que donne de la subsidiarité l'article 3 B du traité de Maastricht inverse dans une large mesure l'esprit qui commande l'enseignement en la matière de l'Église catholique, en ce que la "Communauté » y occupe une place qui paraît plus centrale que résiduelle. Elle n'interdit pas une certaine centralisation au profit des instances européennes au nom d'une meilleure réalisation des « objectifs assignés » ainsi que des « dimensions ou des effets de l'action envisagée »; aux termes de cet article, « la Communauté agit dans les limites des compétences qui lui sont conférées et des objectifs qui lui sont assignés. Dans les domaines qui ne relèvent pas de sa compétence exclusive, la Communauté n'intervient, conformément au principe de subsidiarité, que si et dans la mesure où les objectifs de l'action envisagée ne peuvent pas être réalisés de manière suffisante par les États membres et peuvent donc, en raison des dimensions ou des effets de l'action envisagée, être mieux réalisés au niveau communautaire. Aucune action de la Communauté n'excède ce qui est nécessaire pour atteindre les objectifs du présent traité ».

9. René Capitant, Principes du droit public, Les Cours de droit, 1956-1957, p. 46 (cité par Yves PALAU, "La médiation sociale, une construction idéologique ", Études, décembre 1996, p. 717).

10. Pierre Bigo, La doctrine sociale de l'Église : recherche et dialogue, Paris, PUF, 1965, p. 280 . 
sociaux »; ces derniers se sont largement recrutés parmi les militants issus et/ou encore membres des Églises chrétiennes - catholique ou protestantes -; beaucoup ont transité par le Parti socialiste unifié (PSU) ou le mouvement communautaire (par exemple, La Vie nouvelle, animée par Philippe Warnier). Une bonne traduction des enjeux proprement politiques est fournie par le débat qu'organise la Nouvelle revue socialiste en janvier 1979 : le clivage s'y avère particulièrement net entre Jean-Pierre Chevènement et Michel Rocard. Le fondateur du CERES se fait méfiant vis-à-vis d'une remise en cause du rôle central de l'État : «Derrière le thème de l'expérimentation sociale se cache l'opposition de la société civile et de l'État, qui me paraît une fausse fenêtre. En tant que socialiste, je considère en effet que l'opposition fondamentale n'est pas l'opposition de l'État et de la société civile, mais tout simplement la lutte des classes ». (...) Il me semble (...) que "le thème de l'expérimentation sociale, comme moyen privilégié de changement social, fait l'impasse sur la nécessité de la lutte politique pour conquérir l'État, le transformer fondamentalement ${ }^{11}$. La stratégie défendue par Michel Rocard est diamétralement opposée : "L'expérimentation sociale c'est tout ce qui fait que les luttes politiques, et notamment les luttes des classes (...) ne sont pas seulement des thèmes de motion ou des slogans nationaux ». (...) L'État est-il l'agent principal et l'organisateur de la transformation sociale ? Eh bien, oui, il $\mathrm{y}$ a ici un énorme problème. "Je tiens, moi, l'État actuel comme le frein principal de la transformation sociale ${ }^{12}$. Dans cette perspective, il n'est pas indifférent que deux anciens Premiers ministres - Raymond Barre et... Michel Rocard co-signent, en 2002, la préface de l'ouvrage de Jean-François Six et Véronique Mussaud, La médiation (Seuil), et reconnaissent dans la médiation la "fille de la fraternité ", qui permet de concilier l'exercice de la liberté et celle de l'égalité.

Dans cette perspective de la défense du rôle quasi inaliénable des corps intermédiaires, les associations occupent une position qui est apparue de plus en plus importante au cours des dernières décennies. Considérées comme un rempart contre l'autoritarisme et le totalitarisme et comme un vecteur de socialisation, les associations contribuent à la constitution du tissu conjonctif de la démocratie pluraliste ; ce faisant, elles garantissent activement la liberté des citoyens. On entend ici l'écho de la position défendue par Tocqueville sur fond d'observation de la jeune démocratie états-unienne : les associations ont avant tout pour fonction de faire écran entre l'État démocratique et le citoyen et de protéger celui-ci contre toute velléité de despotisme, velléité proportionnelle au degré d'égalité des conditions sociales : "Pour que les hommes restent civilisés ou le deviennent, il faut que parmi eux l'art de s'associer se développe et se perfectionne dans le même rapport que l'égalité des conditions s'accroît ${ }^{13}$. Le Cercle Jean-Moulin exprime

11. Nouvelle Revue Socialiste, janvier $1979, \mathrm{n}^{\circ} 37$, p. 8.

12. Ibid., p. 14.

13. Alexis De TocQueville, De la démocratie en Amérique, Paris, UGE, 1963, p. 123 ; 282. 
cette même idée au début des années 1960 : «Le citoyen ne peut surmonter son impuissance que si, dans chaque domaine, des relais de pouvoir se développent à un niveau où il lui soit possible de participer » ${ }^{14}$. Pour souligner le rôle spécifique et central qu'assume l'association, Jean-François Six observe que la médiation a acquis droit de cité en deux vagues : la première est liée à la mise en place par le ministère du Travail d'un système de médiation pour les conflits sociaux et à l'instauration d'un mode de règlement des litiges des citoyens avec l'administration, sous la forme d'un médiateur de la République. La seconde vague, que l'auteur situe vers la fin des années 1970, " a accompagné le raz-de-marée du mouvement associatif : des personnes ont commencé à demander à des associations, proches de leur vie de tous les jours, des actes de médiation. Et ceci dans tous les domaines. (...) Les associations sont bien souvent "médiation" elles-mêmes » ${ }^{15}$. Le journal La Croix s'était alors fortement investi dans une réflexion sur la place des associations dans la société française ; il avait constitué un dossier substantiel comportant les résultats d'un sondage sur la participation des Français à la vie associative (29 novembre 1975) et une série de quatre articles sur des expériences locales de la vie associative (14-17 janvier 1976) ; s'y était ultérieurement ajoutée une longue interview de M. Taittinger, secrétaire d'État auprès du ministre de l'Intérieur (9 juillet 1976). Plus récemment, dans son éditorial qui introduit au dossier que publie la revue Projet à l'occasion du centenaire de la loi de 1901 sur les associations, le jésuite Pierre Martinot-Lagarde souligne que l'affirmation d'une liberté fondamentale comme celle de s'associer renvoie à un fondement anthropologique : les associations traduisent la recherche de réponses collectives à des sollicitations toujours nouvelles; dès lors, il convient de «se réjouir (...) d'une malléabilité qui permet à l'individu de passer de la frontière du privé au public, une frontière que la pression de la subjectivité contemporaine, le souci de soi ou la fragilité institutionnelle rendent bien malaisée à franchir ${ }^{16}$.

Notons à cet égard que les trois éléments qu'Yves Palau retient comme constitutifs de la construction idéologique qui fonde la technique de la médiation sociale sont en fait trois facettes ou trois dimensions d'une même transformation générale des rapports État-société : l'exigence d'une société civile, comme sphère autonome entre l'État et l'individu; l'idéal participatif, comme revitalisation de l'espace démocratique; la valorisation de l'association, acteur principal d'un nouveau jeu social. Y. Palau estime que sans ces trois éléments, qui donnent à la médiation sociale sa dimension politique en l'inscrivant dans un courant théorique alternatif de la tradition républicaine et qui sont une réponse à la crise de la représentation, la médiation apparaitrait comme simple technique de gestion des conflits. En tant que pratique constitutive d'un espace intermédiaire entre public et privé, la médiation se fonde en définitive sur les valeurs de «l'hétérogénéité,

14. Club Jean-Moulin, L’État et le citoyen, Paris, Seuil, 1961, p. 199.

15. Jean-François SIX, "Médiation et médiateurs ", Études, juillet-août 1993, p. $45 ; 53$.

16. Pierre Martinot-Lagarde, «L'utopie associative ", Projet, hiver 2000-2001, p. 34. 
de la diversité, du particulier, voire du désordre, en lieu et place de l'idéologie républicaine, plus encline à prendre en compte le général, l'ordre et l'homogénéité. ${ }^{17}$ Dans ce domaine de l'analyse des formes du pouvoir, Michel Wievorka lie explicitement les débats sur la médiation à ceux sur la gouvernance, considérés comme deux dimensions certes différentes mais complémentaires de la réflexion sur les enjeux institutionnels de la démocratie, de l'organisation sociale et du processus décisionnel, singulièrement dans le cadre de la politique de la ville ${ }^{18}$.

L'Église s'applique-t-elle à elle-même les principes qu'elle édicte à l'intention de la société politique ? Cette transposition est en fait des plus limitées : le pape Paul VI déclare ainsi en 1969 que le principe de subsidiarité, appliqué à l'Église, "ne doit pas être confondu avec une prétendue requête de "pluralisme" qui toucherait la foi, la loi morale et les lignes fondamentales des sacrements »; c'est dire que l'unité doctrinale et disciplinaire prime sur la liberté. La vérité ne se divise ni ne se négocie. Ce n'est pas là le moindre des paradoxes d'une institution dont le jésuite Paul Valadier estime, sur un ton critique, que "si elle mettait réellement en jeu la collégialité et la synodalité qui lui sont essentielles, ainsi que la subsidiarité, on verrait alors qu'elle pourrait honorer certains éléments démocratiques et rejoindre, par là, l'attente légitime des fidèles » ${ }^{19}$. Il n'est pas sans intérêt de constater que la réflexion de l'Église catholique du Québec, province canadienne dont on sait le rôle de premier plan qu'elle a joué dans le développement des pratiques de médiation, a posé dès le début des années 1990 des jalons visant à mettre en question l'exercice du pouvoir hiérarchique de l'épiscopat et à reconnaître dans la communauté chrétienne "la matrice et la médiation par excellence de l'éducation de la foi " : "C'est évident, l'éducation de la foi par et dans l'approche communautaire suppose aussi un déplacement des lieux du

17. Yves Palau, «La médiation sociale, une construction idéologique ", Études, décembre 1996, p. 614 ; 618. Voir également Jacques Ion, « Le modèle associatif entre l’idéal démocratique et la nostalgie des corps intermédiaires ", La Revue de l'économie sociale, avril 1988 ; JeanPierre Worms, "Nouveau contexte, nouveaux enjeux pour les associations ", Après-demain, décembre 2001, p. 7-12, qui écrit qu' "entre l'universalisme abstrait de la République et l'incarnation concrète d'une démocratie participative, il manque dans l'espace public les instruments d'une liaison effective. Forger ce chaînon manquant, tel est le défi majeur que les associations doivent relever » (p. 12) ; Évelyne PISIER, «Le besoin de médiation ", L’Express, 7 avril 1989, p. 52-53, qui constate que "la démocratie représentative n'est jamais assez représentative et, donc, jamais assez démocratique », et qui plaide pour qu'une autre médiation entre gouvernés et gouvernants naisse de confrontations libérées des insuffisances de la politique.

18. Michel Wievorka, "Introduction ", in Michel Wievorka, dir., La médiation, une comparaison européenne, Paris, Éditions de la DIV, 2002, p. 28.

19. Paul Valadier, "Quelle démocratie dans l'Église ?", Études, février 1998, p. 229. Chantal Million-Delsol note à ce propos qu'il " apparaît rapidement à quel point une autonomie, même relative, des communautés de base pourrait remettre en cause l'existence de cette société particulière qu'est l'Église. Érigée en vue d'une finalité précise et autour de dogmes qui ne souffrent aucune critique venue de l'intérieur, elle ne saurait laisser à ses membres actifs une autonomie réelle sans se détruire elle-même" (Le principe de subsidiarité, Paris, PUF [coll. «Que Sais-je ? "], 1993, p. 52-53). 
savoir-faire, du savoir-penser et, peut-être même, du savoir-prier, puisqu'il s'agit de remettre progressivement aux communautés elles-mêmes la responsabilité de leur recherche spirituelle et de leur engagement évangélique ${ }^{20}$.

\section{La fonction médiatrice du ré-investissement de l'espace public par les acteurs religieux}

On ne saurait être outre mesure surpris d'observer que la réflexion développée par l'Église catholique, dans le champ de la théologie morale, sur le « bon gouvernement » et sur les formes que devraient prendre les relations entre l'État et le citoyen traduit aussi pour elle-même, dans la conjoncture actuelle, une volonté de reconsidérer le processus de sécularisation. Aujourd'hui plus que naguère, en effet, les pratiques sociales qui relèvent de la médiation ou qui s'y apparentent représentent pour l'Église, singulièrement pour la place qu'elle occupe ou qu'elle escompte (ré-)occuper dans l'espace public, un véritable enjeu : un enjeu pastoral et religieux, à l'évidence, mais aussi et dans le même mouvement un enjeu proprement politique, si l'on entend par là le désir de participer à la recomposition des relations État-société civile et d'en tirer profit. Les représentants de l'État ne sont d'ailleurs pas indifférents à ce mode d'investissement, moins d'ailleurs pour des raisons qui relèveraient d'une improbable révision du principe de laïcité et de la loi de Séparation des Églises et de l'État que pour des motifs qui tiennent aux capacités de "contrôle et de pacification des relations sociales " et de gestion "plus consensuelle des conflits » ${ }^{21}$. Ministre de l'Intérieur et des Cultes dans le gouvernement Raffarin, Nicolas Sarkozy, qui s'est d'ailleurs fortement investi dans la création du Conseil français du culte musulman, ne déroge pas à la règle : «Mon intérêt pour la religion vient de ma conviction qu'elle peut jouer un rôle dans l'équilibre d'une société. Il ne faut pas oublier le rôle civique qu'a joué l'Église catholique au début du siècle passé. Elle a formé quantité de jeunes citoyens. Hélas, on ne retrouve plus l'équivalent aujourd'hui. Dans la France des banlieues, il y a peu d'églises, peu de mosquées, très peu de temples et de synagogues, donc plus personne pour jouer ce rôle de lien social " ${ }^{22}$. De ce type d'enjeu et des projets de formation qu'il suscite, on dispose d'un bon exemple avec le DESS Management interculturel et médiation religieuse qu'offre l'Institut d'Études Politiques d'Aix-en-Provence. L'une des options de ce diplôme précise qu' "à la confluence des champs de recherche intéressant la science politique et la théologie des religions, la médiation et le dialogue interreligieux constituent des méthodes d'avenir dans la gestion pacifique des tensions culturelles et cultuelles.

20. Comité conseil formé par le Comité exécutif de l'Assemblée des évêques du Québec, "La médiation communautaire dans l'éducation de la foi ", L’Église canadienne, 26, n 5, $1^{\text {er }}$ avril 1993, p. 141.

21. Jean-Pierre Bonafé-Schmitt, "Avant-propos ", in La médiation, art. cit., p. 7.

22. Nicolas SARKOZY, "La laïcité n'est pas l'adversaire de la religion ", entretien avec Henri TincQ, Le monde des religions, $\mathrm{n}^{\circ}$ 1, sept.-oct. 2003, p. 68. 
Ils supposent des réseaux au-delà des logiques confessionnelles, ethniques et éthiques, en cours de légitimation ou de constitution. Cette option s'intéresse au "discours" de ce dialogue et à sa mise en pratique. Cette science de la médiation et du dialogue a pour fonction essentielle de participer au maintien de la paix sociale et internationale à une époque de pluralisme culturel-cultuel à la fois local et mondial ».

La sollicitation des uns rencontre ainsi la sollicitude des autres... On peut d'ailleurs se demander si, pour certains de ses responsables institutionnels, l'Église catholique ne se perçoit pas non seulement comme l'inspiratrice mais aussi comme un possible archétype ou un modèle de corps intermédiaires, voire comme le corps intermédiaire par excellence. Sur ce plan de la révision stratégique, un texte officiel de l'Église de France fait figure de véritable manifeste : le rapport présenté par le Père Gérard Defois, alors secrétaire général de l'épiscopat et aujourd'hui archevêque de Lille, à l'occasion de l'Assemblée plénière de l'épiscopat à Lourdes en 1981. La version originale de ce rapport s'intitule : Perspectives missionnaires. Réflexions historiques et sociologiques. Propositions d'action. L'une des lignes directrices de ce texte vise à disqualifier une vision plus militante que proprement institutionnelle de la pastorale catholique et de la place de l'Église dans la société : "L'opposition militants/pratiquants a joué comme un conflit d'interprétation et donc de pouvoir dans l'Église en France. Sans négliger l'apport prophétique de militants qui ont porté le fer de lance d'une responsabilité éthique de l'Église en notre société, il nous revient de remarquer les ambiguités quant au témoignage et à la communion dans l'importation en terre chrétienne de ce modèle d'action sociale " ${ }^{23}$. Ces " ambiguïtés " imposent une révision radicale de la stratégie pastorale, qui s'exprime sous la forme de cinq propositions d'action : - "de la mission-présence à la mission-communication »;

- «toute avancée missionnaire passe par une reconnaissance de l'identité chrétienne»;

- "assurer le "service missionnaire de la transcendance" en ce monde est une tâche missionnaire pour aujourd'hui »;

- «la mission c'est faire exister socialement l'Église »;

- " toute mission est universelle".

Arrêtons-nous à la quatrième de ces propositions, dont la teneur se trouve en forte congruence avec le thème de la médiation. Sous la plume de Gérard Defois, elle est l'occasion de dresser un bilan - globalement négatif - de la stratégie d'effacement volontaire conduite par les forces vives d'une Église qui entendait, dans la ligne du second concile du Vatican, promouvoir une institution «servante et pauvre». Rappelons d'un mot le contexte politique national dans lequel se

23. Gérard Defors, "La mission dans la société et l'histoire ", in L'Église que Dieu envoie (Assemblée plénière de l'épiscopat, Lourdes 1981), Paris, Le Centurion, 1982, p. 84. 
trouve inscrite cette proposition : à la suite des élections présidentielle et législatives du printemps 1981, le Parti socialiste est maître, depuis quelques mois, du pouvoir exécutif. L'un des engagements de campagne de François Mitterrand, devenu chef de l'État, porte sur la constitution d'un "grand service public unifié et laïque d'éducation », sans contrainte ni spoliation. L'enseignement privé, qui se situe presque en totalité dans la mouvance de l'Église catholique, se trouve ainsi placé au premier rang des cibles du gouvernement Mauroy. Dans sa négociation avec Alain Savary, ministre de l'Éducation nationale, la position du Secrétariat national de l'enseignement catholique (SNEC), dirigé par le Père Guiberteau, est principalement fondé sur la mise en valeur de l'utilité sociale de l'enseignement catholique : l'argument s'appuie notamment sur la souplesse de structures largement décentralisées et, par là, la capacité d'innovation et d'expérimentation des établissements privés. En d'autres termes, l'enseignement public aurait plus à perdre qu'à gagner à absorber un " concurrent » dans lequel il convient surtout de voir un émulateur et un pionnier. Face à l'État, c'est une fois encore le rôle des corps intermédiaires qui est mis en avant, comme une condition des libertés. Dans un article du Monde intitulé «La richesse d'une nation ", le Père Guiberteau écrit quelques mois avant l'échec du projet Savary que « face aux États modernes dont les moyens d'une toute-puissance sont une menace contre les libertés concrètes des citoyens, apparaît le rôle des "groupes partiels", communautés exprimant des héritages et des valeurs. (...) La nation attend de nous que nous soyons différents et c'est par là que nous pensons pouvoir rendre service à la jeunesse » ${ }^{24}$. Ce qui est alors nouveau, c'est moins la capacité de résistance, traditionnellement bien rôdée, de l'enseignement catholique que cette version modernisée de son argumentaire et, surtout, l'attention croissante et pour une part inédite qu'y prête désormais l'épiscopat. En effet, dix ou quinze ans plus tôt, dans la mouvance du Concile, les évêques ne se seraient sans doute pas battus outre mesure pour défendre un secteur d'activité qui pouvait paraître doublement illégitime : d'une part, du fait de la responsabilité " naturelle » de l'État en matière d'éducation et donc du caractère de simple suppléance de la fonction exercée par l'Église en ce domaine ; d'autre part, en raison du risque de contre-témoignage que pouvait constituer un enseignement catholique dominé par les catégories sociales privilégiées. À court terme, l'enjeu est dès lors de définir, prioritairement en direction de l'épiscopat, une position qui fasse droit à la défense du dispositif élaboré par la loi Debré du 31 décembre 1959 et qui soit à la fois, ad intra, institutionnellement légitime et, ad extra, politiquement acceptable. À plus long terme, l'objectif est de fonder sur un réexamen des relations religion-système social un nouveau discours et de nouvelles pratiques.

La position que défend Gérard Defois est ainsi tout entière dictée par la volonté de réviser de façon radicale la stratégie jusqu'alors adoptée par la hiérarchie

24. Paul Guiberteau, « La richesse d'une nation », Le Monde, 3 mars 1984. 
catholique sur la place de l'Église dans l'espace public ${ }^{25}$ : « Il y a quelques années, nous avons prôné l'effacement de l'Église de la place publique. Les institutions chrétiennes, qu'elles soient hospitalières, éducatives ou caritatives, nous paraissaient un obstacle à l'annonce de l'Évangile. Celui-ci devait, au mieux, rassembler les hommes en mouvements spirituels; les organisations et leurs pouvoirs avaient pour nous des relents de chrétienté. Il en est résulté des disqualifications à l'encontre des paroisses et des institutions qui pratiquaient encore ces formes visibles des confusions d'hier entre le social et le chrétien. ${ }^{26}$

Reste alors à proposer une lecture des enjeux politico-religieux qui sauvegarde une place originale à l'Église catholique au nom de son utilité sociale. À cet égard, le Secrétaire général de l'épiscopat présente l'Église comme une sorte de corps intermédiaire ordonné à un certain salut social : l'Église se doit de reconnaître sa propre capacité à restaurer le lien social et à constituer, somme toute, un vecteur original de «médiation » :

En ce monde l'Église peut être le sacrement de la guérison du corps social. Ceci concerne son ministère de communion dans les différences sociales et historiques. C'est la révélation d'une forme autre de la solidarité humaine. Si elle n'est pas un pur reflet du monde, cette Église du Christ doit offrir des lieux de parole et d'échange pour signifier la possibilité d'une fraternité dans la foi, d'une réconciliation reçue en salut parce que le Christ et son Esprit "travaillent» les relations entre les hommes. (...) L'existence sociale et publique d'institutions de communion et de communication témoigne que l'Église en tant que signe du salut est médiatrice de relations humaines et annonce la guérison en Dieu d'un corps social éclaté. Par là, le sacrement du salut révèle sa fécondité sociale ${ }^{27}$.

"Faire exister socialement l'Église ", cela doit ainsi conduire à engager une double série d'actions : faire prendre conscience aux acteurs religieux eux-mêmes de leur capacité à s'inscrire de façon originale et efficace dans le tissage et l'entretien du lien social; amener les partenaires potentiels de l'Église à reconnaître son savoir-faire spécifique dans le traitement des conflits, dans la gestion des effets de l'exclusion et, plus largement, dans l'organisation des rapports sociaux (paroisses, associations à caractère éducatif, caritatif ou spirituel, etc.).

Peut-on parler à proprement parler de résultats directs de ce changement de stratégie, ou, plutôt, de simples effets de conjoncture ? Le partage entre ces deux lectures également possibles est évidemment difficile à opérer : quelle est la part de la volonté délibérée, et quelle est celle d'une logique sociale dénuée de tout caractère intentionnel ? À tout le moins peut-on dire que le rapport Defois de 1981 établit de façon déterminée un plan d'action, mais la mise en œuvre et

25. Sur la portée et les incidences, entendues plus largement, de ce changement de stratégie, voir: Jacques PALARD, «Les mutations du militantisme catholique en France : la dimension politique des ruptures institutionnelles ", Revue internationale de politique comparée, vol. 7, $\mathrm{n}^{\circ} 2,2000$, p. 385-414.

26. Gérard DefoIs, «La mission dans la société et l'histoire ", art. cit., p. 97.

27. Ibid., p. 98-99. 
l'efficience de celui-ci dépend tout autant sinon plus des transformations du système sociopolitique que de la qualité d'une analyse et d'une argumentation qui se trouvent d'ailleurs énoncées à un moment où elles sont à la fois pensables et recevables. Le disciple de Pierre Bourdieu que fut Gérard Defois pourrait probablement avaliser pareille interprétation en termes de détermination relative des pratiques sociales, y compris discursives.

En tout état de cause, la place de l'institution religieuse dans la société française ainsi que ses modes opératoires ont notablement changé. La re-territorialisation de la pastorale catholique, qui s'est opérée à compter du début des années 1980 au profit de la paroisse et du diocèse et au détriment des mouvements d'action catholique, est à ranger au rang des changements majeurs. Ce passage tendanciel d'un mode de gestion sectoriel - qui se sera révélé relativement éphémère - à un mode de gestion territorial est porteur d'effets non seulement internes à l'Église mais également sociétaux : ainsi, c'est le cadre paroissial qu'une équipe de chercheurs de Rouen a retenu pour étudier des opérations de développement social des quartiers. Cette équipe a pris la " décision problématique » de "traiter la paroisse, son périmètre, les initiateurs des "orientations politiques", ses "opérateurs", mandatés ou associés, ses "populations-cibles", ses équipements, ses actions, etc., avec la même grille de lecture que celle qu'on adopterait pour analyser territoire, opérateurs et action de toute organisation ${ }^{28}$. Elle a adopté une approche qui permet d'analyser les modes d'implication et d'intervention sociales d'acteurs religieux dans l'espace public local. Cette approche a été reconduite et élargie lorsque l'étude s'est par la suite étendue aux associations musulmanes. Une attention particulière a alors été portée aux relations entre acteurs religieux et acteurs politiques et, plus précisément, au mode d'instrumentalisation politique du religieux, à des fins de régulation sociale:

Au commencement, les relations du père F. avec les pouvoirs communaux de droite puis de gauche sont difficiles car les élus sont méfiants vis-à-vis des associations à caractère confessionnel qu'il anime. En effet, il revendique non seulement la dimension chrétienne mais aussi l'alliance de la foi chrétienne et musulmane pour favoriser la régulation chrétienne. (...) Néanmoins, les systèmes politico-institutionnels prenant conscience que la socialisation religieuse, qu'elle soit chrétienne ou musulmane, est un moyen possible de favoriser des comportements normés au sein d'une jeunesse largement considérée comme manquant de repères, la Ville, l’État et la région financent l'association Amitié Chrétiens Musulmans dans le cadre du contrat de ville alors même que l'intitulé de l'action comporte une référence religieuse : «Regarder l'autre comme quelqu'un qui est comme toi : créé par Dieu ${ }^{29}$.

28. Claude Legrand, Philippe Le Moigne, Cité des citoyens et communauté paroissiale. Catholicisme et nouvelles formes de gestion de l'espace public dans les quartiers de développement social, Rouen, Institut de Développement social, Laboratoire d'Étude et de Recherche sociales, 1995 , p. 10.

29. Guido DE RIDDER, Manuel BouChER, Mohamed BELgASMI, Nouvelles solidarités et nouvelles concurrences. Acteurs publics, associations musulmanes et catholiques dans les quartiers, Canteleu, Laboratoire d’Études et de Recherche sociales, Institut de développement social, 2000, p. 45-46. 
À un niveau d'appréhension plus large, Jean-Luc Brunin estime, dans son étude de «l'Église des banlieues », que "l’Église joue son rôle de médiation en permettant aux chrétiens d'avancer dans la voie de la solidarité avec les étrangers, les blessés de la vie, les oubliés de la croissance économique. (...) Elle s’y engage comme témoignage rendu à la puissance de la victoire du Christ ressuscité qui a fait tomber le mur de la haine entre les hommes, et a fait advenir une authentique fraternité universelle. ${ }^{30}$ On perçoit dans cette herméneutique de l'agir chrétien l'étroite liaison entre une lecture religieuse du social et une lecture sociale du religieux. Dans cette même perspective, lors du Forum organisé par les Fils de la Charité à Saint-Ouen en octobre 1997 sur la présence et l'action des chrétiens dans les banlieues, l'accent a été mis notamment sur la proximité ${ }^{31}$, dans la ligne de l'incarnation, ainsi que sur la gestion de l'interculturalité, dans la double perspective de la catholicité et de l'expérimentation ${ }^{32}$.

En 1997, dans leur Lettre aux catholiques de France, les évêques établiront un lien étroit entre la reconnaissance par l'Église de la laïcité comme composante de l'histoire nationale et la revendication de la participation pleine et entière des catholiques à la vie du pays : "Nous tenons à être reconnus non seulement comme des héritiers, solidaires d'une histoire nationale et religieuse, mais aussi comme des citoyens qui prennent part à la vie actuelle de la société française, qui en respectent la laïcité constitutive et qui désirent y manifester la vitalité de leur foi ${ }^{33}$.

30. Jean-Luc BRUNIN, L'Église des banlieues. L’urbanité: quel défi pour les chrétiens?, Paris, Éditions de l'Atelier, 1998, p. 116 ; sur l'évolution de la dimension religieuse de l'action sociale, voir Gilbert VINCENT, dir., La place des œuvres et des acteurs religieux dans les dispositifs de protection sociale. De la charité à la solidarité, Paris-Montréal, L'Harmattan, 1997.

31. Cette notion de proximité, qui imprègne de plus en plus les discours politiques, est en forte affinité avec celle de médiation; le maire de Charleroi présente ainsi le colloque qui se tient dans sa ville sur Médiation de quartier... De l'esprit à l'évaluation, en novembre 2003 : «En proposant un cadre de fonctionnement original et professionnel basé sur la dynamique du volontariat et de la coopération, la médiation de quartier a véritablement investi le terrain qu'on lui destinait, celui de la proximité. (...) Outil de construction et de réparation de la relation, elle est utile non seulement au niveau interpersonnel mais aussi dans les rapports sociaux. (...) Elle rend la citoyenneté à l'individu » (brochure de présentation du colloque).

32. Les principaux documents de ce forum sont présentés dans Documents Épiscopat (Bulletin du secrétariat de la Conférence des évêques de France), n² 2-3, février 1998. La gestion en 1988 par Michel Rocard, nouveau Premier ministre, de la crise néo-calédonienne fait explicitement appel à des représentants des Églises chrétiennes en vue de renouer le dialogue entre les diverses communautés : "Dans ce processus, le médiateur n'intervient pas de l'extérieur ; au contraire, il s'implique personnellement, en tant qu'acteur social lui-même " (Danièle HERvIEULÉGER, "The Past in the Present: Redefining Laïcité in Multicultural France ", in Peter L. BERGER, dir., The Limits of Social Conflict. Conflict and Mediation in Pluralist Societies, op. cit., p. 79). La mission à Bagdad, en septembre 2004, d'une délégation du Conseil français du culte musulman destinée à contribuer à la libération de deux journalistes français retenus en otages relève d'une logique analogue.

33. Mgr Claude Dagens, Proposer la foi dans la société actuelle. Lettre aux catholiques de France, Paris, Cerf, 1997, p. 28. Ce texte, rédigé par Mgr Dagens, a été adopté par l'Assemblée plénière des évêques de France en novembre 1996 ; il résulte d'un long processus de consultation au sein des diocèses et d'une collaboration entre évêques et théologiens qui n'ont pas d'équivalent, en France, dans la production des textes épiscopaux. 
Sur le plan international également, l'Église a joué à de nombreuses reprises un rôle de médiation et de bons offices, sans rendre toutefois, à strictement parler, d'arbitrage dans un différend international ${ }^{34}$. La perte de sa puissance politique, avec celle des États pontificaux, conditionne pour ainsi dire sa crédibilité. La position défendue par le socialiste Léon Blum, en 1945, au sortir de la Deuxième Guerre mondiale, est à cet égard on ne peut plus significative ; évoquant la constitution d'un Corps international, il envisage, à côté des États, l'opportunité de ce qu'il appelle "une autre présence » :

C'est à la Cour de Rome que je pense, au Saint-Siège apostolique. Sa participation au même titre que celle des États serait par elle-même le signe le plus éclatant que, dans l'univers de demain, d'autres puissances compteront que les puissances temporelles. Sa coopération active permettrait de hausser sur un plan supérieur et de régler par des « concordats " généraux toutes ces catégories de litiges avec les États, qui, à l'intérieur du cadre national, altèrent la vie politique et conduisent à d'insupportables conflits. Ce rôle conviendrait assurément à une Église qui est pacifique par essence, puisqu'elle incarne une religion de paix et qui l'est aussi par fonction, si je puis dire, puisque sa constitution même est d'ordre international ${ }^{35}$.

L'activité nationale et internationale de médiation de l'Église demeure d'actualité. Au Chiapas, l’Église, et au premier chef Mgr Ruiz Garcia, évêque de San Cristobal de las Casas, représente un agent politique actif qui a conduit les protagonistes vers la négociation et la pacification: elle y est intervenue à la fois dans la formation et l'émergence de nouveaux acteurs et dans les processus de conciliation entre les zapatistes et le gouvernement fédéral mexicain ${ }^{36}$. La communauté Sant'Egidio, qui est née d'une initiative d'Andrea Riccardi dans les banlieues pauvres de Rome, où elle continue son action, est un groupe de pression efficace auprès du Vatican ; elle met en œuvre une active "diplomatie parallèle» qui associe engagements religieux, caritatif et international. On sait le rôle que la communauté, que d'aucuns appellent "l'ONU du Trastevere ", a joué au Mozambique, au Guatemala, dans la rédaction de la plate-forme de Rome sur la paix en Algérie, ou, à la demande de Jean-Paul II, dans le dialogue interreligieux ${ }^{37}$.

Le développement de ce dialogue interreligieux, par la prise en compte de la diversité et de l'altérité qu'il induit, contribue à transformer la conception de l'évangélisation et du salut traditionnellement défendue par l'Église catholique, et donc également de la médiation institutionnelle dans l'économie du salut. Cette

34. Cf. Jean-Marc TicchI, Aux frontières de la paix. Bons offices, médiations, arbitrages du Saint-Siège (1878-1922), Rome, École française de Rome, 2002, p. 12-13.

35. Léon Blum, À l'échelle humaine, Paris, Gallimard, 1971 (1945), p. 155-156.

36. Cf. Pilar Gil TÉBAR, «Entre la médiation politique et la recomposition sociale : l'Église catholique dans le conflit du Chiapas ", Archives de sciences sociales des religions, 1997, n 97, p. 63-72.

37. Cf. Philippe Leymarie, "Une communauté religieuse dans la médiation des conflits. Les bâtisseurs de paix de Sant'Egidio ", Le Monde diplomatique, septembre 2000. 
conception, qui a imprégné les perspectives théologiques du concile Vatican II, est fondée sur une approche "inclusiviste » : les religions non chrétiennes témoignent d'une authentique quête de Dieu, mais le christianisme est présenté comme l'espace de leur plein accomplissement, même s'il ne prétend plus être le passage obligé vers le salut (position défendue par les tenants de l'"exclusivisme»). Elle pose donc comme des limites structurelles au dialogue interreligieux, limites qu'entend dépasser le courant "pluraliste » : celui-ci cherche à reconnaître la richesse intrinsèque des diverses religions et, par là, à rendre obsolète et à disqualifier le sentiment de supériorité que le christianisme peut donner de lui-même auprès de ses interlocuteurs non chrétiens ${ }^{38}$. L'affirmation des raisons communes et le choix délibéré de la reconnaissance des valeurs de l'autre donnent forme à un référentiel qui fonde à son tour la place de l'institution catholique comme instance de dialogue dénuée de toute volonté de puissance.

Est-il besoin de le préciser, cette analyse a délibérément adopté une approche fonctionnelle et non substantive de la religion. L'objectif est en effet de comprendre, dans une optique de sociologie de la connaissance, comment des référents culturels peuvent induire des modalités d'action et des préférences politiques. Il importait, pour se faire, d'opérer une forme de " concordance des temps ». Comme l'exprime Régis Debray dans son rapport sur l'enseignement du fait religieux à l'école, «l'histoire des religions peut prendre sa pleine pertinence éducative, comme moyen de raccorder le court au long terme, en retrouvant les enchaînements, les engendrements longs propres à l'humanitude ${ }^{39}$.

Dans les manifestations concrètes des pratiques de médiation conduites par des acteurs catholiques - ou, plus largement, de culture chrétienne -, qu'est-ce qui relève, d'une part, du structurel religieux et, d'autre part, du conjoncturel et de stratégies sociopolitiques ? En d'autres termes, est-on fondé à parler d'une commune matrice, dont les productions seraient singulières sans cesser pour autant de présenter entre elles plus qu'un air de parenté : une même " grammaire » et une même pragmatique ? Une base interprétative s'avère ici centrale : la question de la médiation a fondamentalement à voir avec les rapports de pouvoir : pouvoir dans le fonctionnement propre de l'institution religieuse et pouvoir dans les rapports entre sphère religieuse et sphère politique. Dans Le temps des médiateurs, paru en 1990 et dans lequel d'aucuns ont vu «l'ouvrage de référence qui

38. Voir André Fossion, "Comme chrétiens, comment comprendre la pluralité des religions? ", Cahiers Paraboles, n 8, 2000, p. 7-27: "Pour dépasser l'exclusivisme et aller audelà de l'inclusivisme, le chrétien a-t-il peut-être encore à apprendre à penser l'absolu dont il se réclame comme un absolu relationnel» (p.27). Sur cette question, voir également Jean DupuIs, Vers une théologie chrétienne du pluralisme religieux, Paris, Cerf (coll. "Cogitatio fidei »), 1997 ; Monique ÆBISCHER-CRETTOL, Vers un cecuménisme interreligieux. Jalons pour une théologie chrétienne du pluralisme, Paris, Cerf (coll. "Cogitatio fidei »), 2001.

39. Régis Debray, L'enseignement du fait religieux dans l'École lä̈que, Rapport au ministre de l'Éducation nationale, février 2002. 
a marqué la "décennie de la médiation" " ${ }^{40}$, l'auteur, qui est prêtre ${ }^{41}$, invite à "cesser de donner comme définition de la médiation - pour séduire - ce sophisme-slogan : "Pas de gagnant, pas de perdant". Nous disons au contraire, à ceux qui demandent une médiation : "Vous serez tous les deux perdants, vous aurez chacun à abandonner quelque chose, mais ceci afin d'être tous les deux gagnants. Il s'agit d'un passage par une certaine mort à vous-même, à votre souveraineté, pour entrer dans une nouvelle naissance et créer une nouvelle relation" " ${ }^{42}$.

L'analyse de la médiation est justiciable d'approches complémentaires, notamment anthropologique et politologique. Elle est en outre indissociable d'autres modes de transformation sociale et institutionnelle, dont relèvent en particulier la décentralisation des systèmes politiques et le développement des associations, chacune de ces évolutions mettant en jeu à la fois les missions de l'État, la place du citoyen et l'organisation des rapports d'autorité et de légitimité entre l'un et l'autre. Le développement des pratiques de médiation manifeste en effet un fonctionnement social moins hiérarchique et plus réticulaire, moins centralisé et plus segmenté, ainsi que de nouvelles modalités de structuration des identités collectives et individuelles. Vincent de Briant et Yves Palau soulignent que la « dimension instrumentale des nouvelles médiations ne rend pas pour autant illégitime l'analyse de leur nature idéologique. Elle est même rendue d'autant plus nécessaire que la médiation porte sur une manière d'être en société et d'être présent au monde parmi les plus emblématiques de notre civilisation qui (...) remet en cause les fondements les plus sûrs de ce qui avait constitué sa modernité ${ }^{43}$.

40. Michèle Guillaume-Hofnung, "Le concept de médiation ", communication au colloque Médiation-Éducation et Droits de l'homme. Pour vivre ensemble en ville, Paris, ministère de la Ville, UNESCO, mars 2000.

41. Ordonné prêtre en 1956, Jean-François Six devient en 1960 directeur au Grand séminaire de la Mission de France. Il oriente dès lors son action et sa réflexion dans le domaine des relations avec les non-croyants : il est successivement consultant au Secrétariat pour les noncroyants au Concile Vatican II, responsable, en 1966, du Secrétariat français pour les noncroyants et fondateur, en 1976, de la Revue Brèche. En 1980, il crée l'Association Droits de l'homme et solidarité. Il dirige depuis 1987 l'Institut de formation à la médiation et préside le Centre national de la médiation.

42. Jean-François SIX, "Médiation et médiateurs", Études, juillet-août 1993, p. 50. On pourrait également se reporter au numéro 18 des Cahiers de la médiation (2 $2^{\mathrm{e}}$ trimestre 2003) dans lequel J.-F. Six signe un article intitulé " "Empowerment" ? » (p. 1-21) qui s'inspire très largement d'une communication présentée par une conférencière à un séminaire de formation des Équipes Saint-Vincent, association catholique d'entraide sociale. L'auteur rappelle que le Conseil de cette organisation, qui se réfère à l'esprit de saint Vincent de Paul, a pris comme objectif pour 2002-2005: "Construire une relation d'égal à égal ", thématique qui se décline ainsi : "Se situer en partenaire de la personne en difficulté ; oser une remise en question personnelle dans nos relations avec les autres; reconnaître à chacun la capacité de se développer et de trouver en lui-même ses ressources et ses solutions ".

43. Vincent de Briant, Yves Palau, La médiation. Définition, pratiques et perspectives, Paris, Nathan, 1999, p. 115. 
Il va sans dire que l'institution catholique ne vise pas à tirer parti de cette crise de la modernité pour (re)conquérir une position centrale au sein de la société française. Elle n'en cherche pas moins à s'inscrire dans un mouvement porteur qui paraît donner crédit, y compris au sein de la classe politique, à l'utilité sociale des confessions religieuses. Plus largement, certains de ses savoir-faire ou certaines composantes de sa doctrine sociale retrouvent une valeur opératoire au moment où se poursuit une recomposition des appareils politiques et administratifs et que la voix citoyenne suscite à nouveau attention et intérêt. Dans ce contexte, où un évêque plaide "la chance d'un christianisme fragile " ${ }^{44}$, une fraction active des catholiques tend à défendre moins une Église instituée qu'une Église instituante. On comprend dès lors que certains d'entre eux se soient trouvés aux avantpostes dans la promotion de nouvelles pratiques en syntonie avec leur conception des rapports sociaux et de la subsidiarité.

Jacques PALARD CERVL - Institut d'Études Politiques de Bordeaux

44. Albert RouEt (évêque de Poitiers), La Chance d'un christianisme fragile. Entretiens avec Yves de Gentil-Baichis, Paris, Bayard, 2001. 
Mots-clés : médiation, catholicisme, subsidiarité, espace public, régulation sociale

\section{Résumé}

Le développement des pratiques sociales de médiation dans des secteurs aussi différents que la famille, la justice, l'école, les quartiers urbains... conduit à s'interroger sur le contexte idéologique et institutionnel dans lequel s'inscrit ce type d'innovations. Cette étude cherche à comprendre comment des référents culturels et des orientations religieuses des acteurs catholiques peuvent induire de nouvelles modalités d'action et des préférences politiques spécifiques. Deux voies sont inventoriées: la place du principe de subsidiarité et des corps intermédiaires dans la doctrine sociale de l'Église catholique, et l'exercice d'une fonction médiatrice au travers du ré-investissement de l'espace public par des acteurs religieux. Chacune de ces deux dimensions oriente vers une interprétation en termes d'utilité sociale des confessions religieuses et de prégnance de la matrice catholique en période de crise relative des modes de régulation des rapports sociaux par les institutions politiques et administratives.

\section{Abstract}

The development of social mediation practices in fields as diverse as the family, justice, education, disadvantaged neighborhoods, raises important questions regarding the ideological and institutional framework of such innovations. The present study is an attempt to understand how some of the cultural values and religious orientations of Catholic actors may induce new modes of action and particular political preferences. Two elements are investigated: first, the role of the principle of subsidiarity and of mediating structures in the social doctrine of the Catholic Church; second, the exercise of a mediating function through a reinvestment of religious actors in the public sphere. Each of these two elements lead to an interpretation in term of the social utility of religious groups and of the importance of the Catholic matrix in times of relative crisis of the process of regulation of social relationships by the political and state institutions.

\section{Resumen}

El desarrollo de las prácticas sociales de mediación en sectores tan diferentes como la familia, la justicia, la escuela, los barrios urbanos conduce a interrogarnos sobre el contexto ideológico e institucional en el que se inscribe este tipo de innovaciones. Este estudio busca comprender cómo los referentes culturales y las orientaciones religiosas de los actores católicos pueden inducir nuevas modalidades de acción y de preferencias politicas especificas. Dos vías son inventariadas : el lugar del principio de subsidiaridad y de los cuerpos intermedios en la doctrina social de la Iglesia católica, y el ejercicio de una función mediadora a través de la reinversión del espacio público por actores religiosos. Cada una de estas dos dimensiones orienta hacia una interpretación en términos de utilidad social de las confesiones religiosas y de potencialidad de la matriz católica en períodos de crisis relativa de los modos de regulación de las relaciones sociales por las instituciones politicas y administrativas. 